\title{
Vergleichbare Leistungsfähigkeit wie Komposite
}

Ein Schwerpunkt der modernen Zahnmedizin liegt auf der minimalinvasiven Entfernung von Zahnhartsubstanz und der Anwendung adhäsiver Füllungsmaterialien. Gleichzeitig steigt die Nachfrage nach zahnfarbenen Versorgungen weltweit. Restaurationsmaterialen wie Komposite und Glasionomerzemente (GIZ) kommen diesen Entwicklungen entgegen - wie beispielsweise das mikrogefüllte Hybrid-Komposite Gradia Direct Posterior und das Glasionomer-basierte Füllungskonzept EQUIA (beide GC). Die eindrucksvollen 4-Jahres-Ergebnisse der im Folgenden vorgestellten Studie bestätigen das Leistungsvermögen des EQUIASystems im Vergleich mit dem Komposit.

Glasionomere werden bereits seit geraumer Zeit erfolgreich in der Zahnmedizin angewendet. Sie punkten insbesondere mit Eigenschaften wie der Adhäsion auch in feuchtem Milieu, thermischer Kompa- tibilität zum Zahnschmelz und einer guten Biokompatibilität [Ilie N et al. Clin Oral Investig. 2012; 16: 489-98]. Hinzu kommen antikariogene Eigenschaften, die durch die Fluoridfreisetzung bewirkt wer- den. Im Vergleich zu herkömmlichen Glasionomeren verfügen moderne hochvisköse GIZ über verbesserte physikalische Eigenschaften [Bonifacio CC et al. Acta Odontol Scand. 2012; 70: 471-477]. Das Restaurationssystem EQUIA besitzt den Anspruch, die genannten Vorteile und Verbesserungen mit denen eines Komposit-Coatings zu kombinieren. Dazu wird die Glasionomer-basierte Füllungskomponente (EQUIA Fil) mit einem nanogefüllten, lichthärtenden Lack (EQUIA Coat) überzogen. Wie sich dieses Füllungskonzept nun im Vergleich zu einem Komposit in der klinischen Praxis über einen Zeitraum von 5 Jahren behauptet, war Gegenstand der nachfolgend beschriebenen Studie. Präsentiert werden die Ergebnisse nach 4 Jahren.

\section{Klinisches Leistungsvermögen von EQUIA im Vergleich zu Komposit}

Untersucht wurde in der vorliegenden Invivo-Studie das EQUIA-System - bestehend aus den genannten Komponenten EQUIA Fil und EQUIA Coat - im Vergleich zu dem Seitenzahn-Komposit Gradia Direct Posterior (GC) in Kombination mit dem selbstätzenden Adhäsiv G-Bond (GC) 

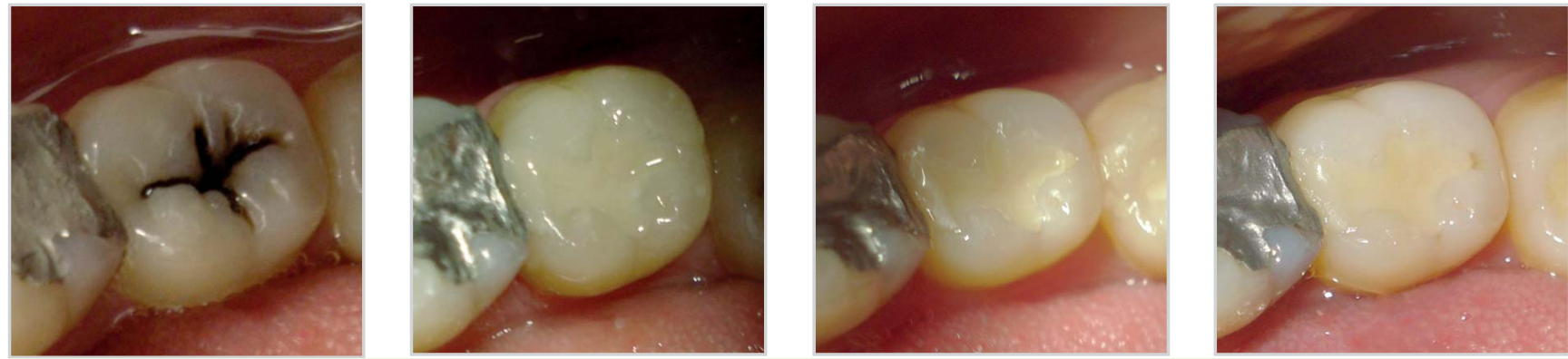

Abb. 1 EQUIA Klasse-II-Füllungen nach 1, 2, 3 und 4 Jahren (v. I. n. r.) (Quelle: Prof. S. Gurgan).

[Gurgan S et al. Oper Dent. 2015; 40: 134143]. Durchgeführt wurde die Untersuchung in der zahnärztlichen Fakultät, Abteilung Restaurative Zahnmedizin an der Hacettepe Universität Ankara (TR). Bei 59 Patienten mit einem Durchschnittsalter von 24 Jahren wurden 140 posteriore Läsionen bei Ober- und Unterkiefermolaren und Prämolaren (80 Klasse-I- und 60 Klasse-II-Läsionen) mit EQUIA oder Gradia Direct Posterior versorgt. 2 erfahrende Anwender legten unter Beachtung der Herstelleranweisungen die Restaurationen; 2 unabhängige Experten bewerteten die Ergebnisse nach 12, 24, 36 und 48 Monaten gemäß der modifizierten US Public Health Service-Kriterien (USPHS) und mithilfe von PVS-Abdrücken (Polyvinylsiloxan) und Negativ-Repliken qualitativ unter dem Rasterelektronenmikroskop (REM). Die statistischen Analysen wurden mit dem McNemar-Test, dem Chi-QuadratTest nach Pearson und dem Cochran QTest $(p<0,05)$ erhoben.

Von den zu Beginn der Studie angetretenen 59 Studienteilnehmern verblieben 52 nach 4 Jahren (Recall-Rate: 88,1\%); die Versorgung der übrigen 7 Patienten konnte nicht bewertet werden, da sie verzogen waren. Insgesamt konnten 126 Restaurationen (76 Klasse-I- und 50 Klasse-II-Restaurationen) evaluiert werden. Die Ergebnisse sind überzeugend: Bei Versorgungen mit Gradia Direct Posterior lag die Erfolgsrate sowohl bei Klasse-I- als auch bei Klasse-II-Läsionen bei 100 \%. Für EQUIA lag sie für Klasse-I-Restaurationen ebenfalls bei $100 \%$ und bei Klasse-II-Versorgungen bei 92,7 \% (einzig eine Klasse-IIRestauration musste als Folge einer Randfraktur nach 3 und eine weitere nach 4 Jahren ausgetauscht werden). Es wurden bei beiden Restaurationsmaterialien sig- nifikante Unterschiede in der marginalen Adaptation und Verfärbung nach 48 Monaten im Vergleich zur Ausgangssituation sowohl für Klasse-I- als auch Klasse-II-Restaurationen gefunden $(p<0,05)$. Keine Versorgung zeigte Verschlechterungen bezüglich der anatomischen Form, Farbübereinstimmung, Sekundärkaries, postoperativen Sensibilität, Oberflächenstruktur und Haftung $(p>0,05)$; die rasterelektronenmikroskopischen Auswertungen bestätigten diese klinischen Befunde ( $\bullet$ Abb. 1). EQUIA verfügt laut den Ergebnissen nach 4 Jahren über eine vergleichbare klinische Leistungsfähigkeit im Seitenzahnbereich wie das Komposit Gradia Direct Posterior.

\section{Weitere Studien bestätigen Potenzial von EQUIA \\ $\nabla$}

Auch weitere Studien bezeugen die klinische Performance von EQUIA: Diem et al. untersuchten Fuji IX GP Extra (EQUIA Fil) mit und ohne Applikation des nanogefüllten Komposit-Lacks G-Coat Plus (EQUIA Coat) im Vergleich mit einem mikro-gefüllten Hybrid-Komposit [Clin Oral Investig. 2014; 18: 753-759]. Nach 3 Jahren konstatierten die Forscher, dass der Auftrag des Lacks eine geringere Abrasion der okklusalen Kavitäten bewirkte. Darüber hinaus läuft derzeit eine 5-Jahres-Studie von Klinke et al.; die fortgeschrittenen Zwischenergebnisse der Wissenschaftler nach 48 Monaten sind vielversprechend [Biffar R et al. Abstract Nr. 0039, ConsEuro 2015]. Bei einer generellen Betrachtung der Materialgruppe empfehlen darüber hinaus die Ergebnisse eines empirischen Studien-Reviews eine Neubewertung von Glasionomerzementen in der modernen Zahnheilkunde, da unbedarft-indirekte
Vergleiche der klinischen Eigenschaften hochvisköser GIZ mit Alternativen (wie beispielsweise Amalgamfüllungen) auf unkontrollierten klinischen Longitudinalstudien basieren - und diese die Argumente für die üblichen (negativen) klinischen Empfehlungen für Glasionomerzemente verstärkt haben könnten [Mickenautsch S et al. PLOS One 2013; 8: e78397]. Dies wirft ein neues Licht nicht nur auf GIZ, sondern auch auf die Verwendung von zahnärztlichem Amalgam. Aufgrund der schwindenden Patientenakzeptanz lässt sich ohnehin der Trend feststellen, auf dieses Restaurationsmaterial zu verzichten, und auch globale Bestrebungen könnten den Einsatz von Amalgam in der Zahnmedizin beeinflussen. Beispielsweise sieht die Minamata-Konvention aufgrund des Quecksilbergehaltes von ca. $50 \%$ eine schrittweise Reduzierung ("phase-down") von Dentalamalgam vor [United Nations Environment Programme. Press release: 19 January, 2013. 'Minamata' Convention Agreed by Nations. Global Mercury Agreement to Lift Health Threats from Lives of Millions World-Wide. Online: http://www.unep.org/newscentre/ default.aspx? DocumentID=2702\&Article ID=9373]. Diese Entwicklungen unterstreichen die Relevanz weiterer Erforschungen moderner, adhäsiver Materialien wie beispielsweise Komposite und Glasionomere.

\section{S Gurgan, ZB Kutuk, E Ergin, SS Oztas, FY Cakir}

\section{Korrespondenzadresse}

Prof. Dr. Sevil Gurgan

Hacettepe University - School of Dentistry 06100 Ankara, Türkei

sgurgan@hacettepe.edu.tr

Diese Veröffentlichung wird unterstützt von der GC Germany GmbH, Bad Homburg 